\title{
Vertical abundance stratification in the blue horizontal branch star HD 135485
}

\author{
V. R. Khalack ${ }^{1,2}$, F. LeBlanc ${ }^{1}$, D. Bohlender ${ }^{3}$, G. A. Wade ${ }^{2}$, and B. B. Behr ${ }^{4, \star \star \star}$ \\ 1 Département de Physique et d'Astronomie, Université de Moncton, Moncton, N.-B., E1A 3E9, Canada \\ e-mail: khalakv@umoncton.ca \\ 2 Department of Physics, Royal Military College of Canada, PO Box 17000 stn 'FORCES', Kingston, Ontario, K7K 4B4, Canada \\ 3 National Research Council of Canada, Herzberg Institute of Astrophysics, 5071 West Saanich Road, Victoria, BC, V9E 2E7, Canada \\ 4 Department of Astronomy, University of Texas at Austin, 1 University Station C1400, Austin TX 78712-0259, USA
}

Received 26 October 2006 / Accepted 29 January 2007

\section{ABSTRACT}

\begin{abstract}
Context. It is commonly believed that the observed overabundances of many chemical species relative to the expected cluster metallicity in blue horizontal branch (BHB) stars appear as a result of atomic diffusion in the photosphere. The slow rotation of BHB stars (with $T_{\text {eff }}>11500 \mathrm{~K}$ ), typically $v \sin i<10 \mathrm{~km} \mathrm{~s}^{-1}$, is consistent with this idea.

Aims. In this work we search for observational evidence of vertical chemical stratification in the atmosphere of HD 135485. If this evidence exists, it will demonstrate the importance of atomic diffusion processes in the atmospheres of BHB stars.

Methods. We undertake an extensive abundance stratification analysis of the atmosphere of HD 135485, based on recently acquired high resolution and S/N CFHT ESPaDOnS spectra and a McDonald-CE spectrum.

Results. Our numerical simulations show that nitrogen and sulfur reveal signatures of vertical abundance stratification in the stellar atmosphere. It appears that the abundances of these elements increase toward the upper atmosphere. This fact cannot be explained by the influence of microturbulent velocity, because oxygen, carbon, neon, argon, titanium and chromium do not show similar behavior and their abundances remain constant throughout the atmosphere. It seems that the iron abundance may increase marginally toward the lower atmosphere. This is the first demonstration of vertical abundance stratification of metals in a BHB star.
\end{abstract}

Key words. stars: atmospheres - stars: horizontal-branch - stars: chemically peculiar - stars: individual: HD 135485

\section{Introduction}

HD 135485 appears to be an evolved star that lies close to or on the horizontal branch (Trundle et al. 2001). It has mid-B spectral type and shows many prominent absorption lines of metallic species in its spectrum. The most recent absolute and differential abundance analysis of HD 135485 was performed by Trundle et al. (2001). They found a large (0.5-1.0 dex) enhancement of metal abundances in comparison with the solar composition. Similar results were obtained previously by Schönberner (1973) and Dufton (1973) from analysis of moderate-resolution photographic spectra. In an attempt to explain the anomalous chemical composition in the atmosphere of HD 135485, Dufton (1973) considered the possibility of binary system evolution, where the mass flow onto HD 135485 from a high mass component may have created the observed enhancements of metal species. In order to try to prove this hypothesis, Dufton investigated the radial

* Based on observations made with ESPaDOnS at the CanadaFrance-Hawaii Telescope (CFHT) operated by the National Research Council (NRC) of Canada, the Institut des Sciences de l'Univers of the Centre National de la Recherche Scientifique (CNRS) and the University of Hawaii and on observations made with Echelle Spectrograph on the McDonald Observatory 2.1-m Otto Struve Telescope.

$\star \star$ Full Table 2 is only available in electronic form at http://www . aanda.org

$\star \star \star$ Current address: US Naval Observatory, 3450 Massachusetts Avenue NW, Washington DC 20392, USA. velocity during a five month period, but found no variation (see Trundle et al. 2001, for details).

Another possible explanation proposed for the unusually high metallicity is that the star was formed in a region of high metallicity, such as the Galactic Center, and during the long period of its stellar evolution it could have traveled over a large distance $(\sim 26 \mathrm{kpc})$ as demonstrated by Trundle et al. (2001) from analysis of the kinematical properties of HD 135485.

Together with general enrichment of all metals (except nickel which is significantly depleted) with respect to hydrogen, Trundle et al. (2001) have also found a slightly enhanced helium abundance of $\sim 0.3$ dex. They interpret this fact along with the anomalously high $(\sim 1.3 \mathrm{dex})$ nitrogen overabundance as an indication that the carbon-nitrogen $(\mathrm{CN})$ cycle took place during the hydrogen-burning phase on the main sequence. The relatively normal carbon enhancement might reflect a balance between its being destroyed by the $\mathrm{CN}$ cycle and created via helium core flashes during the red giant phase (which could also explain the observed neon enrichment in the atmosphere). These facts and the present position of HD 135485 on the HertzsprungRussell (HR) diagram argue that this star is evolved and probably belongs to the blue horizontal branch (BHB) stars. The observed low (projected) rotational velocity $\left(v \sin i<4 \mathrm{~km} \mathrm{~s}^{-1}\right)$ of HD 135485, the observed chemical peculiarities (except the helium enrichment) and the effective temperature $T_{\text {eff }}=15500 \mathrm{~K}$ (Trundle et al. 2001) are typical for BHB stars.

Comprehensive surveys of BHB star abundances show that the stars hotter than $T_{\text {eff }} \simeq 11500 \mathrm{~K}$ have abundance anomalies 
Table 1. Journal of spectroscopic observations of HD 135485.

\begin{tabular}{ccccccc}
\hline \hline $\begin{array}{c}\text { Date } \\
(\mathrm{UT})\end{array}$ & $\begin{array}{c}\text { HJD } \\
(2450000+)\end{array}$ & Instrument & $\begin{array}{c}t_{\mathrm{exp}} \\
(\mathrm{s})\end{array}$ & $\begin{array}{c}S / N \text { ratio } \\
(\text { pixel })^{-1}\end{array}$ & $\begin{array}{c}\text { Coverage } \\
\lambda(\AA)\end{array}$ & Resolution \\
\hline 2001 Mar. 09 & 1978.9242 & $\mathrm{CE}$ & 600 & 67 & $4760-5570$ & 60000 \\
2005 May 19 & 3509.8677 & ESPaDOnS & 600 & 150 & $3720-10290$ & 80000 \\
2005 May 19 & 3509.8753 & ESPaDOnS & 600 & 140 & $3720-10290$ & 80000 \\
\hline
\end{tabular}

when compared to the BHB stars of lower effective temperature in the same cluster ${ }^{1}$ (Glaspey et al. 1989, Grundhal et al. 1999). Also, Peterson et al. (1995); Behr et al. (2000) and Recio-Blanco et al. (2004) showed the existence of a discontinuity in stellar rotation velocity distribution of BHB stars at $T_{\text {eff }} \simeq 11500 \mathrm{~K}$. All of the hotter stars show modest rotation $\left(v \sin i<10 \mathrm{~km} \mathrm{~s}^{-1}\right)$, while the cooler stars are rotating more rapidly.

Other observational results show both photometric jumps (Grundhal et al. 1999) and photometric gaps (Ferraro et al. 1998), also occurring at $T_{\text {eff }} \simeq 11500 \mathrm{~K}$, as well as low measured surface gravities. The low gravity, abundance anomalies and slow rotation suggest that microscopic atomic diffusion is effective in stellar atmospheres of the BHB stars with $T_{\text {eff }} \geq$ $11500 \mathrm{~K}$. In this picture, the competition between radiative acceleration and gravitational settling yields a net acceleration on atoms, which results in their diffusion through the atmosphere. A consequence of this process is vertically-stratified abundances of chemical elements. The aim of this paper is to attempt to detect observationally such vertical stratification of elements in the atmospheres of BHB stars. Direct measurement of this stratification from line profile analysis would provide a convincing argument in favour of efficient chemical diffusion in the atmospheres of BHB stars with $T_{\text {eff }} \geq 11500 \mathrm{~K}$.

The most important difference between HD 135485 and most BHB stars is that it has an enhanced helium abundance. Therefore HD 135485 might be a very young BHB star in which helium has not yet settled. However, a detailed investigation of this possibility is outside the scope of this paper.

To verify if the abundances of some chemical species are vertically stratified in the atmosphere of HD 135485, we undertook a detailed analysis of available high-resolution spectra employing a modification of the approach for abundance stratification analysis developed by Ryabchikova et al. (2005). In Sect. 2 we discuss the properties of the observed spectra, and in Sect. 3 we describe the atmospheric parameters of HD 135485 and modelling details. The results of abundance determinations are considered in Sect. 4, while a description of vertical stratification for some chemical species is shown in Sect. 5. A discussion of our results follows in Sect. 6.

\section{Observations}

Spectroscopic observations of HD 135485 were undertaken in March 2001 and May 2005. The journal of spectroscopic observations is shown in Table 1, where individual columns give the UT date of the observation, heliocentric Julian Date of the observation, the instrument specification, exposure time, signal-to-noise $(\mathrm{S} / \mathrm{N})$ ratios per pixel (the peak typically occurs around $5400 \AA$ ), spectral coverage and resolving power (which depends on central wavelength).

\footnotetext{
${ }^{1}$ Most of the known BHB stars are found in globular clusters.
}

\subsection{McDonald Cassegrain Echelle spectrum}

The first spectrum of HD 135485 (from Table 1) was obtained in the framework of a study of the rotational characteristics $(v \sin i)$ of BHB stars (Behr 2003) using the Cassegrain Echelle (CE) Spectrograph (McCarthy et al. 1993) on the McDonald Observatory 2.1-m Otto Struve Telescope. This instrument provides a nominal resolving power of $R \sim 60000$. The signalto-noise ratio (per pixel) was computed from the reduced spectrum by smoothing the spectral regions identified as continuum, and then computing the rms deviation between the observed and smoothed spectra.

The package of routines developed by McCarthy (1990) for the FIGARO data analysis package (Shortridge 1993) was employed to reduce the spectrum. The detailed description of the reduction procedure for this particular spectrum, including the wavelength calibration routine and the continuum normalization, is provided by Behr (2003). The procedure follows a standard prescription, with bias subtraction, flat-fielding, order extraction, and wavelength calibration from thorium-argon arc lamp observations. Cosmic ray hits were identified and removed by hand for maximum spectral quality. No smoothing was applied to the spectra.

\subsection{CFHT-ESPaDOnS spectra}

The other two spectra were obtained using the new ESPaDOnS (Echelle SpectroPolarimetric Device for Observations of Stars) spectropolarimeter at the Canada-France-Hawaii Telescope (CFHT). ESPaDOnS is fundamentally similar in construction to the MuSiCoS spectropolarimeter (Donati et al. 1999) and allows the acquisition of an essentially continuous spectrum throughout the spectral range $3700 \AA$ to $10500 \AA$ in a single exposure. The optical characteristics of the spectrograph as well as the spectropolarimeter observing procedures, are described by Donati et al. (in preparation) ${ }^{2}$. Observations were performed in the spectroscopic "sky only" mode of the instrument that provides the highest resolving power $(R \sim 80000)$. This resolution corresponds to velocity elements of $3.75 \mathrm{~km} \mathrm{~s}^{-1}$.

The ESPaDOnS spectra were reduced using the LibreESpRIT reduction tool (Donati et al., in preparation), which is the most recent release of ESpRIT (Donati et al. 1997). The continuum of each spectral order was normalized by fitting a 3rd to 5th order polynomial (as selected by eye) to regions free of prominent spectral lines and not located immediately at the beginning or at the end of the spectral order.

\section{Line profile simulations}

\subsection{Stellar atmosphere parameters}

The line profile simulations were performed using a Phoenix (Hauschildt et al. 1997) LTE (Local Thermodynamic

\footnotetext{
2 For more details about this instrument, the reader is invited to visit wWw . ast .obs-mip.fr/projects/espadons/espadons.html
} 
Equilibrium) stellar atmosphere model with enhanced $(+1.0$ dex $)$ metallicity. The model is calculated assuming $T_{\text {eff }}=15500 \mathrm{~K}$, and $\log g=4.0$, which according to Trundle et al. (2001) provide the best description of observable line profiles of HD 135485. Those authors adopted a microturbulent velocity of $5 \mathrm{~km} \mathrm{~s}^{-1}$ to provide a constant behavior of abundances over the range of equivalent widths measured for sulfur and nitrogen ions. Behr (2003) has adopted for this star $\xi=1.4 \mathrm{~km} \mathrm{~s}^{-1}$ and obtained $v \sin i=0 \mathrm{~km} \mathrm{~s}^{-1}$.

From our perspective, the physical motivation for adopting a microturbulent velocity is unclear. According to Landstreet (1998), photospheric convection should be very weak at the effective temperatures of BHB stars. Landstreet found that spectroscopically-derived microtubulence had decreased from around $4 \mathrm{~km} \mathrm{~s}^{-1}$ in late A stars with $T_{\text {eff }} \sim 8000 \mathrm{~K}$ to $0.5 \mathrm{~km} \mathrm{~s}^{-1}$ in early A stars with $T_{\text {eff }} \sim 10500 \mathrm{~K}$. In particular, strong microtubulence (e.g. $5 \mathrm{~km} \mathrm{~s}^{-1}$ as adopted by Trundle et al. 2001) should be accompanied by strong line asymmetries, which we do not observe. We therefore realistically expect no detectable microturbulence in HD 135485. Of course, Landstreet's analysis was performed for main sequence stars; we might reasonably expect that BHB stars show some differences, although the atmospheric parameters of HD 135485 are very similar to those of main sequence stars.

Based on this guidance, we have tentatively adopted a microturbulent velocity $0 \mathrm{~km} \mathrm{~s}^{-1}$ for our abundance analysis of HD 135485. Using zero microturbulence in spectral synthesis we estimated stellar rotational velocity $v \sin i=2-3 \mathrm{~km} \mathrm{~s}^{-1}$. This is an important assumption for several reasons. First, because diffusion is a slow process, the mixing which might be implied by the presence of microturbulence could serve to reduce or erase chemical stratification. Also, adoption of microturbulence modifies line strengths, leading to systematic changes in inferred mean abundances as well as abundance trends with line strength. As we shall see in Sect. 5, the validity of the assumption of zero microturbulence can be tested, and it is fully consistent with our spectroscopic observations.

\subsection{Procedure}

All of the available spectra were examined to compose a line list for chemical species that are suitable for abundance and stratification analysis. The line identification is performed using the VALD-2 (Kupka et al. 1999; Ryabchikova et al. 1999) and NIST $^{3}$ (version 3.0.3) line databases (Reader et al. 2002). The same sources are used to specify the atomic data for the selected lines. For Fe II and $\mathrm{Cr}$ II lines we extracted atomic data from Raassen \& Uylings ${ }^{4}$ (1998), while for some Fe II lines we also used the atomic data recently obtained by Fuhr \& Wiese (2006). An example of the first ten lines from the line list selected for the abundance analysis of $\mathrm{N}$ II lines is shown in Table $2^{5}$, where references identify sources for the adopted $\log g f$ values, while the given energy levels are taken mostly from the NIST database.

All line profiles are simulated using the ZEEMAN2 spectrum synthesis code (Landstreet 1988; Wade et al. 2001) assuming Gaussian instrumental profiles. The stability of the ESPaDOnS instrumental profile has been investigated using the calibration spectra of a ThAr quartz lamp. We find the instrumental profile to be stable during the night of observation and to reveal no

\footnotetext{
3 http://physics.nist.gov/PhysRefData/ASD/index.html

4 ftp://ftp.wins.uva.nl/pub/orth

5 Full Table 2 is presented in the electronic edition of the journal. A portion is shown here for guidance and content.
}

Table 2. List of spectral lines used for the abundance analysis.

\begin{tabular}{crccl}
\hline \hline \multicolumn{5}{c}{ N II } \\
\hline$\lambda, \AA$ & $\log g f$ & $E_{i}, \mathrm{~cm}^{-1}$ & $\log \gamma_{\text {rad }}$ & Ref. \\
\hline 4447.030 & 0.228 & 164610.76 & 9.16 & NIST \\
4507.556 & -0.817 & 166678.64 & 9.33 & VALD \\
4601.478 & -0.428 & 148940.17 & 9.23 & VALD \\
4613.868 & -0.665 & 148940.17 & 9.23 & VALD \\
4621.396 & -0.514 & 148940.17 & 9.23 & VALD \\
4630.539 & 0.094 & 149076.52 & 9.15 & VALD \\
4779.722 & -0.587 & 166521.69 & 9.58 & VALD \\
4803.287 & -0.113 & 166678.64 & 9.58 & VALD \\
4987.376 & -0.555 & 168892.21 & 9.34 & VALD \\
4994.367 & -0.069 & 168892.21 & 9.34 & VALD \\
\hline
\end{tabular}

dependence on the intensity of the line profile. The ZEEMAN2 code has been modified (Khalack \& Wade 2006) to allow for an automatic minimization of the model parameters using the downhill simplex method (Press et al. 1992). The relatively poor efficiency of the downhill simplex method, requiring a large number of function evaluations, is a well known problem. Repeating the minimization routine several times in the vicinity of a supposed minimum in the parameter space allows us to check if the method converges to the global minimum.

To search for the presence of abundance stratification, we have used two different methods. In the first method, we estimate the abundance of a chemical element from independent analysis of each line profile. For every layer of the stellar atmosphere model (it contains 50 layers) we calculate the line optical depth $\tau_{\ell}$ in the line core. We suppose that each analyzed profile is formed mainly at $\tau_{\ell}=1$, which corresponds to a particular layer of the stellar atmosphere. Finally, for this layer, the respective continuum optical depth $\tau_{5000}$ is specified. In this way, we have built the scale of optical depths $\tau_{5000}$ to track vertical abundance stratification through the analysis of all the available line profiles for a chosen chemical element.

Three free model parameters (the element's abundance, line radial velocity $V_{\mathrm{r}}$ and $v \sin i$ ) were derived from each line profile using the aforementioned automatic minimization routine. HD 135485 has very sharp absorption lines and even for resolution $R=80000$ a line profile usually consists of only 8 to 15 bins. This leads to the fact that the downhill simplex method fails to converge to the global minimum in approximately 2 to $5 \%$ of analyzed line profiles. This means that the automatic minimization routine remains in the vicinity of a local minimum and results in a higher final value of the $\chi^{2}$-function. Repeating the minimization routine with different starting values of the model parameters usually solves this problem and provides a better fit, which is checked using a few additional runs of the simulation routine. Sometimes, analysis of a line profile results in a radial velocity which differs significantly from the average $V_{\mathrm{r}}=4.2 \mathrm{~km} \mathrm{~s}^{-1}$ (obtained from analysis of all line profiles of the same chemical element and other chemical species). This may be evidence for line misidentifications or inaccurate line wavelengths. Therefore lines with radial velocities which differ more than $1.5 \mathrm{~km} \mathrm{~s}^{-1}$ from the average are not taken into account in the modeling and are not included in Table 2.

In the second method, we only analyzed those lines which appear to be good candidates in the previous approach and consider the two-step stratified abundance model with a linear transition zone (Ryabchikova et al. 2003; Wade et al. 2003) to describe the abundance distribution. In this case, all the selected lines were analyzed simultaneously and in the minimization 
routine we operated with 6 free model parameters: the element's abundance in the deeper layers, the (standard) optical depth $\tau_{1}$ of the transition zone lower (deeper) boundary (where the element's abundance begins its linear increase or decrease), the optical depth $\tau_{2}$ of the transition zone upper boundary, the difference between the abundances at two boundaries, the stellar radial velocity and the $v \sin i$. We note that for the second method the downhill simplex method never failed to reach the global minimum. This means that information stored in all of the analyzed lines, modeled simultaneously, is statistically sufficient to provide a unique solution. To determine the averaged abundances for all of the analyzed chemical species, we simultaneously fit the available line profiles using the same three free model parameters used in the first approach.

Initially, we specify arbitrarily the free model parameters in the range of their possible values and simulate the line profiles. We then compare the simulated profiles with the observed spectra, and calculate the reduced $\chi^{2}$, which we adopt as a measure of the fit quality. The expression for the $\chi^{2}$ function, which reflects the agreement between the simulated profiles $\left(I_{i, \lambda_{j}}\right)$ and observed spectra $\left(I_{i, \lambda_{j}}^{\mathrm{obs}}\right)$ is given by:

$\chi_{I}^{2}=\frac{1}{N_{I}} \sum_{i=1}^{N_{I}} \frac{1}{N_{i}} \sum_{j=1}^{N_{i}}\left(\frac{I_{i, \lambda_{j}}^{\mathrm{obs}}-I_{i, \lambda_{j}}}{\sigma\left[I_{i, \lambda_{j}}^{\mathrm{obs}}\right]}\right)^{2}$,

where $\sigma\left[I_{i, \lambda_{j}}^{\mathrm{obs}}\right]$ corresponds to the measurement errors, $N_{I}$ represents the number of profiles indexed by $i$, while $N_{i}$ is the number of pixels in each analyzed line profile. In the first method of the analysis we used $N_{I}=1$. In fact, the simulated spectra are calculated with a resolution of $0.01 \AA$ and so do not provide a direct coincidence of wavelengths in the simulated and observed spectra. Therefore, during the $\chi^{2}$ function evaluation the simulated spectral intensity at the exact observed wavelength is calculated using a linear interpolation.

In order to evaluate the fit errors (and therefore the uncertainties on the derived free parameters), we calculate deviations of the simulated profiles produced as a result of small variations of each of the free parameters, thus introducing a small shift along one axis in the $\chi^{2}$ hyper-space from the point of the function minimum value. Using this procedure, and taking into account the uncertainties of the observational data and the obtained minimum value of the $\chi^{2}$-function, we can estimate the errors of the best-fit parameters.

\section{Results of abundance analysis}

We have identified in the spectra of HD 135485 most of the lines reported by Trundle et al. (2001). No lines of Mn II and Ni II were detectable in our spectra and these elements were therefore not analysed. It seems that Trundle et al. (2001) have determined the Mn II and Ni II abundances from the much stronger ultraviolet lines of these species. The McDonald-CE spectrum has lower spectral resolution than the ESPaDOnS data and is used mainly to confirm the average abundance obtained with the ESPaDOnS spectra.

A preliminary LTE abundance analysis of helium lines suggests an enhanced helium abundance in HD 135485, and the element also seems to be vertically stratified. The helium overabundance obtained is in good agreement with the results reported by Trundle et al. (2001).

The mean photospheric abundances we derive for HD 135485 are reported in Table 3. The second and third
Table 3. Mean atmospheric abundances $\log \left(N / N_{\text {tot }}\right)$ of HD 135485 with uncertainties equal to the standard deviation of $n$ measured lines.

\begin{tabular}{|c|c|c|c|c|c|}
\hline \multirow[b]{2}{*}{ Ion } & \multicolumn{2}{|c|}{ CFHT-ESPaDOnS } & \multicolumn{2}{|c|}{ "McDonald-CE } & \multirow{2}{*}{$\begin{array}{c}\text { Sun } \\
\log \left(N / N_{\text {tot }}\right)\end{array}$} \\
\hline & $\log \left(N / N_{\mathrm{tot}}\right)$ & $\mathrm{n}$ & $\log \left(N / N_{\text {tot }}\right)$ & $\mathrm{n}$ & \\
\hline He I & $-0.58 \pm 0.16$ & 9 & $-0.54 \pm 0.15$ & 3 & -1.11 \\
\hline C I & $-2.78 \pm 0.17$ & 10 & & & -3.65 \\
\hline C II & $-2.69 \pm 0.14$ & 15 & $-2.88 \pm 0.16$ & 4 & -3.65 \\
\hline $\mathrm{N}_{\mathrm{I}}$ & $-2.60 \pm 0.20$ & 3 & & & -4.26 \\
\hline N II & $-3.00 \pm 0.17$ & 21 & $-3.01 \pm 0.18$ & 9 & -4.26 \\
\hline O I & $-2.82 \pm 0.13$ & 11 & $-2.74 \pm 0.19$ & 2 & -3.38 \\
\hline O II & $-3.28 \pm 0.22$ & 9 & & & -3.38 \\
\hline $\mathrm{Ne} I$ & $-3.22 \pm 0.06$ & 4 & & & -4.20 \\
\hline $\mathrm{NaI}$ & $-4.22 \pm 0.24$ & 2 & & & -5.87 \\
\hline $\operatorname{Mg} I$ & $-3.62 \pm 0.20$ & 1 & & & -4.51 \\
\hline Mg II & $-3.73 \pm 0.12$ & 4 & & & -4.51 \\
\hline $\mathrm{Al}$ II & $-5.32 \pm 0.11$ & 7 & & & -5.67 \\
\hline Al III & $-5.10 \pm 0.09$ & 4 & & & -5.67 \\
\hline Si II & $-4.28 \pm 0.23$ & 9 & $-4.23 \pm 0.11$ & 2 & -4.53 \\
\hline Si III & $-3.96 \pm 0.22$ & 2 & & & -4.53 \\
\hline P II & $-5.81 \pm 0.12$ & 8 & $-5.76 \pm 0.13$ & 3 & -6.68 \\
\hline S II & $-4.43 \pm 0.18$ & 48 & $-4.47 \pm 0.17$ & 21 & -4.90 \\
\hline $\mathrm{Cl}$ II & $-6.35 \pm 0.20$ & 1 & $-6.47 \pm 0.20$ & 1 & -6.54 \\
\hline Ar II & $-5.03 \pm 0.05$ & 4 & $-4.96 \pm 0.19$ & 2 & -5.86 \\
\hline Ti II & $-6.70 \pm 0.04$ & 5 & & & -7.14 \\
\hline Cr II & $-6.01 \pm 0.07$ & 5 & $-5.89 \pm 0.14$ & 2 & -6.40 \\
\hline Fe II & $-4.08 \pm 0.16$ & 63 & $-4.16 \pm 0.18$ & 31 & -4.59 \\
\hline Sr II & $-8.50 \pm 0.20$ & 1 & & & -9.12 \\
\hline
\end{tabular}

columns contain respectively the mean abundance of the chemical species and the number of analyzed line profiles in the ESPaDOnS spectra, while the fourth and fifth columns represent similar results obtained from analysis of the McDonald-CE spectrum. The last column contains the solar atmospheric abundances recalculated from $\log \left(N / N_{\mathrm{H}}\right)$ data (Asplund et al. 2005).

\subsection{Light elements: $C$ to $A r$}

Carbon is enhanced by $\sim 0.9$ dex in comparison with the solar abundance and does not show strong evidence of vertical abundance stratification (see Fig. 1a). Nitrogen appears to be strongly enhanced by $\sim 1.7$ dex for the neutral species and by $\sim 1.3$ dex for the first ion. Unlike $\mathrm{C}$, it shows signatures of vertical abundance stratification (see Fig. 2a). The abundances inferred from singlyionized oxygen is almost the same as its solar value, while the neutral species is enhanced by $\sim 0.5$ dex.

Neon and argon appear to be enhanced respectively by $\sim 1.0$ dex and $\sim 0.8$ dex in comparison with their solar abundances. The sodium abundance is estimated from the analysis 
a)

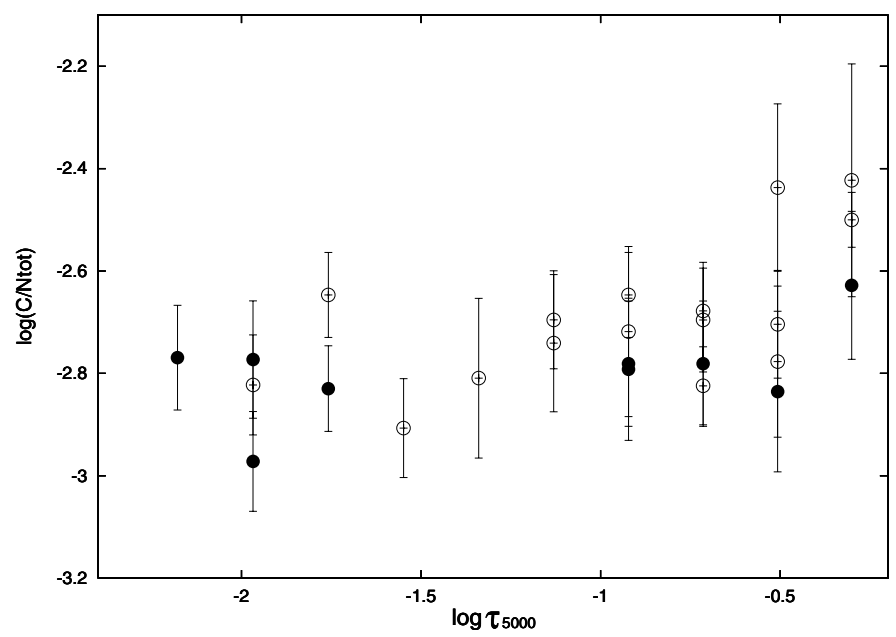

b)

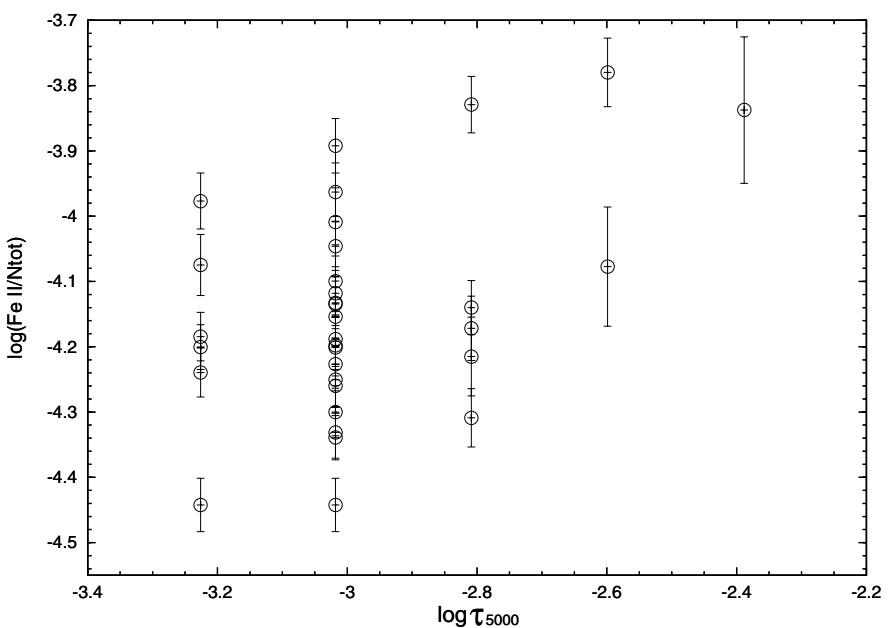

Fig. 1. Abundance estimates from the analysis of a) C I (filled circles) and C II (open circles) and b) Fe II (open circles) line profiles as a function of line (core) formation optical depth. No clear signatures of abundance stratification are visible. The abundances obtained have approximately the same value for all optical depths at which carbon lines are formed.

a)

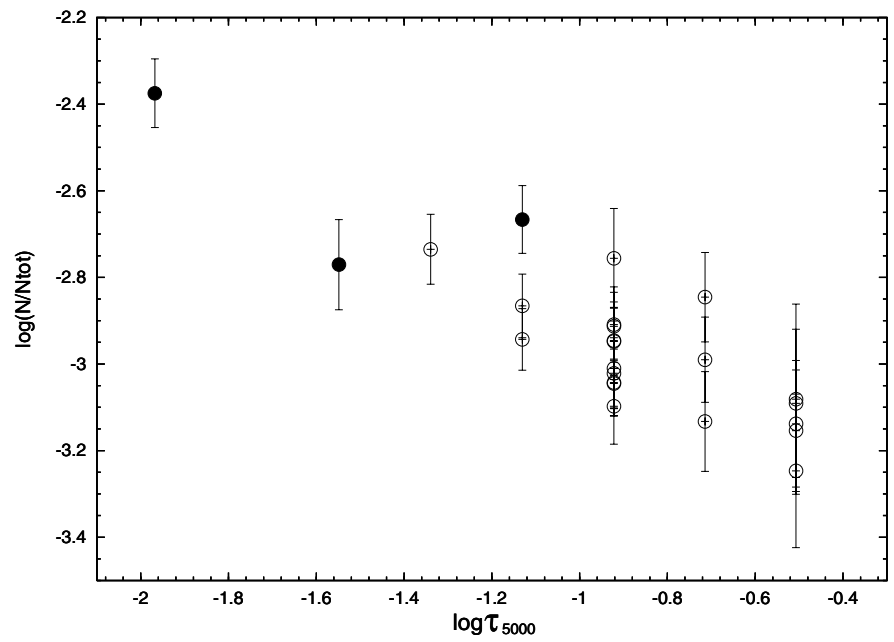

b)

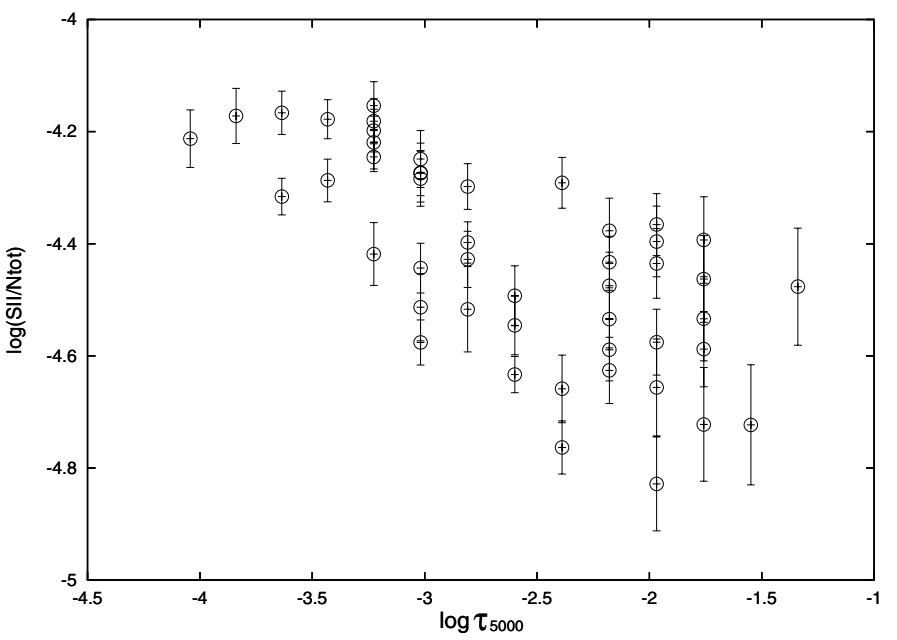

Fig. 2. Same as in Fig. 1 but for a) nitrogen and b) singly ionized sulfur. N I (filled circles) and N II (open circles) lines which are formed in layers with $\log \tau_{5000}<-1.0$ require an enhanced abundance to be fit in comparison with lines of the same ionization stage formed deeper in the atmosphere. A similar situation is found for S II (open circles) lines formed in layers with $\log \tau_{5000}<-1.5$.

of two comparatively weak $\mathrm{NaI}$ lines at $5688.205 \AA$ and $8194.824 \AA$, which results in a $\sim 1.6$ dex enhancement.

Only one unblended MgI line (5172.684 ̊) was found, which results in a magnesium enhancement of $\sim 0.9$ dex. Meanwhile, singly ionized magnesium is enhanced by $\sim 0.8$ dex. Al II and Al III are slightly enhanced by $\sim 0.3$ dex and $\sim 0.5$ dex respectively. Singly ionized silicon has only a $\sim 0.2$ dex enhancement in comparison with its solar abundance, while Si III is enhanced by $\sim 0.6$ dex. Phosphorus shows $\sim 0.9$ dex enhancement. Sulfur appears to be vertically stratified like nitrogen (see Fig. 2b) and its average atmospheric abundance is enhanced by $\sim 0.4$ dex. Chlorine seems to have a solar abundance.

\subsection{Iron-group elements: Ti to Fe}

The spectrum of HD 135485 is extremely rich in Fe II lines. Many of them originate from high-excitation levels with $E_{\mathrm{i}} \geq 10 \mathrm{eV}$. For Fe II lines, we have used mainly
Raassen \& Uylings (1998) results to specify their oscillator strengths and the VALD-2 database to specify the damping coefficients. The Raassen \& Uylings (1998) oscillator strength data usually provides a slightly better agreement between observed and simulated Fe II line profiles than the Kurucz (1993) GFIRON list (Pickering et al. 2001; Ryabchikova et al. 2005). The obtained average atmospheric Fe II abundance is enhanced by $\sim 0.5$ dex and shows some signs of stratification (see Fig. 1b), but in an opposite manner to nitrogen and sulfur (i.e. with the concentration increasing toward deeper layers).

Using the ESPaDOnS spectra we find that chromium and titanium are represented by only a few unblended lines and both elements appear to be enhanced by $\sim 0.4$ dex. The oscillator strengths for Cr II lines have been taken from Raassen \& Uylings (1998), while for Ti II lines we have used VALD-2 database.

We also checked the SrII line at $4077.71 \AA$, which is only marginally visible in these spectra. Its abundance analysis reveals a $\sim 0.6$ dex enhancement - much less than the 
value 1.24 dex enhancement obtained by Trundle et al. (2001). Nevertheless, the enhancements of the other chemical species reported in Table 3 are in good agreement with the results of Trundle et al. (2001).

Adoption of different values of microturbulent velocity by Trundle et al. (2001) and by us should lead to systematic differences in our derived abundance. However, these differences may be reduced because we have used different line lists and atomic data for the abundance analysis. Trundle et al. (2001) have used mostly NIST atomic data for all chemical species, while we have tried to use the most precise atomic data currently available for each element (see Table 2).

\section{Vertical abundance stratification}

Applying the technique described in Sect. 3.2 we have tried to determine if the abundances of some chemical species are vertically stratified. To reach this aim, we need to analyze as many line profiles with different depths of formation (characterised by different excitation potential and line strength) as possible. According to Table 3, a comprehensive analysis can only be performed for carbon, nitrogen, oxygen, sulfur and iron, which are represented in the spectrum of HD 135485 by a sufficient number of lines. In general, we have selected for our analysis lines free of predicted or inferred blends. However, if a blend is from a line of the same chemical element that forms the main line profile, such a line was also included in our simulation.

The very sharp absorption lines of the HD 135485 (8 to 15 bins per line profile), the relatively low signal-to-noise ratio and the uncertainties in the atomic data provide comparatively high errors in abundances inferred from a single line. Taking into account these uncertainties as well as ionization balance errors, we can still see some tendencies of vertical abundance stratification for some of the analyzed chemical elements. Figure 2 shows a systematic trend, wherein $\mathrm{N}$ and $\mathrm{S}$ lines formed higher in the atmosphere provide abundances which are significantly larger than those formed lower in the atmosphere. To check the influence of differences in $\log g f$ values in different databases we have performed independent simulations of sulfur lines using NIST and VALD2 atomic data and have found similar systematic trends in both cases.

The trend observed in the abundances of $\mathrm{N}$ and $\mathrm{S}$ was previously reported by Trundle et al. (2001), but they interpreted it in terms of microturbulence. However, such an interpretation would require a similar behaviour of the lines of $\mathrm{C}, \mathrm{O}$ and $\mathrm{Fe}$, which we do not observe. For example, although the inferred nitrogen and sulfur abundances appear to increase systematically with line strength, carbon and oxygen abundances are approximately constant, whereas the iron abundance shows a weak tendency to decrease with line strength (see Fig. 1). The lack of a coherent trend in the abundances derived from weak versus strong lines in spectra of different elements is not consistent with a microturbulent interpretation, and is fully consistent with chemical stratification. Our simulations of Fe II lines show that adoption of a microturbulent velocity $\xi=2 \mathrm{~km} \mathrm{~s}^{-1}$ results in well visible decreasing of iron abundance with line strength. We point out that this conclusion does not necessarily imply that microturbulence is not present, but rather that it cannot explain the observed systematic abundance trends, that its presence is not implied by any of the data discussed in this paper, and that its presence would be surprising given published observational and theoretical results (Landstreet 1998). Our results therefore suggest that the observed systematic trends in the abundances of $\mathrm{N}$ and S imply that these elements are vertically stratified in the atmosphere of HD 135485.

To obtain an estimate of the abundance distribution as a function of optical depth for $\mathrm{N}$ and $\mathrm{S}$, we have employed a two-zone empirical model with a transition zone (Ryabchikova et al. 2003; Wade et al. 2003) and analyzed simultaneously the same list of lines selected for each element. The final results for nitrogen and sulfur stratification in the atmosphere of HD 135485 are presented in Table 4 and are illustrated in Fig. 3. They are in a good accordance with the abundance stratification data that we have obtained from the first method for $\mathrm{N}$ and $\mathrm{S}$ (see Fig. 2). For comparison, we have also simulated the same list of lines assuming the same abundance of the element (fitted as a free parameter) at different atmospheric depths. From the last two columns in Table 4 we can see that the model with stratified abundance distribution results in a $30 \%$ lower $\chi^{2}$-function than the model with uniform abundance. This fact shows that application of stratified model significantly improves our fit of analysed line profiles.

The $\mathrm{N}$ and $\mathrm{S}$ abundance stratifications were also inferred assuming other stellar atmosphere models with the same gravity $\log g=4.0$, but with the different effective temperatures $T_{\text {eff }}=14500 \mathrm{~K}$ and $T_{\text {eff }}=16500 \mathrm{~K}$, to estimate the influence of effective temperature errors on the vertical abundance stratification profiles. Our simulations show that an effective temperature increase does not effect the final results, while its decrease leads to significant changes of the vertical abundance stratification profile. The stellar atmosphere model with lower temperature results in a steeper stratification profile for $\mathrm{S}$ and an increase in the average $S$ abundance by 0.4 dex, while for nitrogen it provides approximately a 0.8 dex increase in abundances derived from lines formed deeper in the atmosphere where $\log \tau_{5000}>-1.0$. This leads to a reversed stratification profile for $\mathrm{N}$. Therefore the results obtained (see Table 4) for sulfur can be considered to be a lower limit for its abundance stratification profile, whereas a uniform vertical distribution of $\mathrm{N}$ cannot be ruled out.

In conclusion, the analysis of our spectra shows vertical stratification of of $\mathrm{S}$ and possibly $\mathrm{N}$, while the elements $\mathrm{C}, \mathrm{O}, \mathrm{Ne}$, $\mathrm{Ar}, \mathrm{Cr}$ and Ti seem to have an homogeneous abundance throughout the parts of the atmosphere that we can sample. Meanwhile, we cannot draw firm conclusions concerning the stratification of $\mathrm{Fe}, \mathrm{Mg}, \mathrm{Si}, \mathrm{P}$ and $\mathrm{Al}$. Some of these elements are represented by a small number (see Table 3 ) of unblended line profiles and it is hard to obtain clear results.

\section{Discussion}

According to Trundle et al. (2001), HD 135485 is an evolved star that probably belongs to the BHB group. Its slow rotation, high effective temperature $\left(T_{\text {eff }}=15500 \mathrm{~K}\right)$ and observed abundance anomalies (see Table 3 ) support the idea that microscopic atomic diffusion is effective in its atmosphere.

Our analysis suggests that sulfur is moderately overabundant and increases in concentration in the upper atmospheric layers. Nitrogen is strongly overabundant and shows a similar signature of vertical stratification, although the derived stratification of this element depends sensitively on the adopted effective temperature (which is uncertain by about $\pm 1000 \mathrm{~K}$ ). Similar results for these two species were obtained earlier by Trundle et al. (2001), but explained in terms of a microturbulent velocity field. Absence of any abundance stratification for several other elements as well as some hints that the iron abundance might be enhanced in deeper atmospheric layers (i.e. opposite to the tendency shown by $\mathrm{N}$ and $\mathrm{S}$ ) shows that this interpretation is not able to correctly explain the spectroscopic properties of this star. A more plausible 
Table 4. Parameters of the vertical abundance distribution. Here the columns specify respectively the element, its abundance in deeper layers, the abundance difference between the two boundaries, the optical depth $\tau_{1}$ of the transition zone's lower (deeper) boundary, where the element's abundance increases (or decreases) linearly, the optical depth $\tau_{2}$ of the transition zone's upper boundary, the radial velocity and $v \sin i$ with the respective error estimates and the fit quality for the model with abundance stratification $\chi_{\mathrm{s}}^{2}$ and without it $\chi_{\mathrm{n}}^{2}$.

\begin{tabular}{|c|c|c|c|c|c|c|c|c|}
\hline \multirow[t]{2}{*}{ El. } & \multicolumn{2}{|c|}{$\overline{\text { Abundance in } \log \left(N / N_{\text {tot }}\right)}$} & \multicolumn{2}{|c|}{ 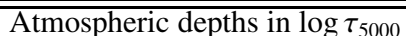 } & \multirow{2}{*}{$\begin{array}{c}V_{\mathrm{r}} \\
\mathrm{km} \mathrm{s}^{-1}\end{array}$} & \multirow{2}{*}{$\begin{array}{l}V \sin i \\
\mathrm{~km} \mathrm{~s}^{-1}\end{array}$} & \multirow[b]{2}{*}{$\chi_{\mathrm{s}}^{2}$} & \multirow[b]{2}{*}{$\chi_{\mathrm{n}}^{2}$} \\
\hline & Low atmosphere & Abundance difference & $\log \tau_{1}$ & $\log \tau_{2}$ & & & & \\
\hline $\mathrm{N}$ & $-3.15 \pm 0.03$ & $0.68 \pm 0.25$ & $-0.67 \pm 0.27$ & $-2.75 \pm 0.45$ & $-4.20 \pm 0.13$ & $1.3 \pm 0.7$ & 2.61 & 3.83 \\
\hline $\mathrm{S}$ & $-4.63 \pm 0.02$ & $0.94 \pm 0.28$ & $-1.31 \pm 0.16$ & $-3.00 \pm 0.40$ & $-4.24 \pm 0.08$ & $3.0 \pm 1.2$ & 6.87 & 10.06 \\
\hline
\end{tabular}

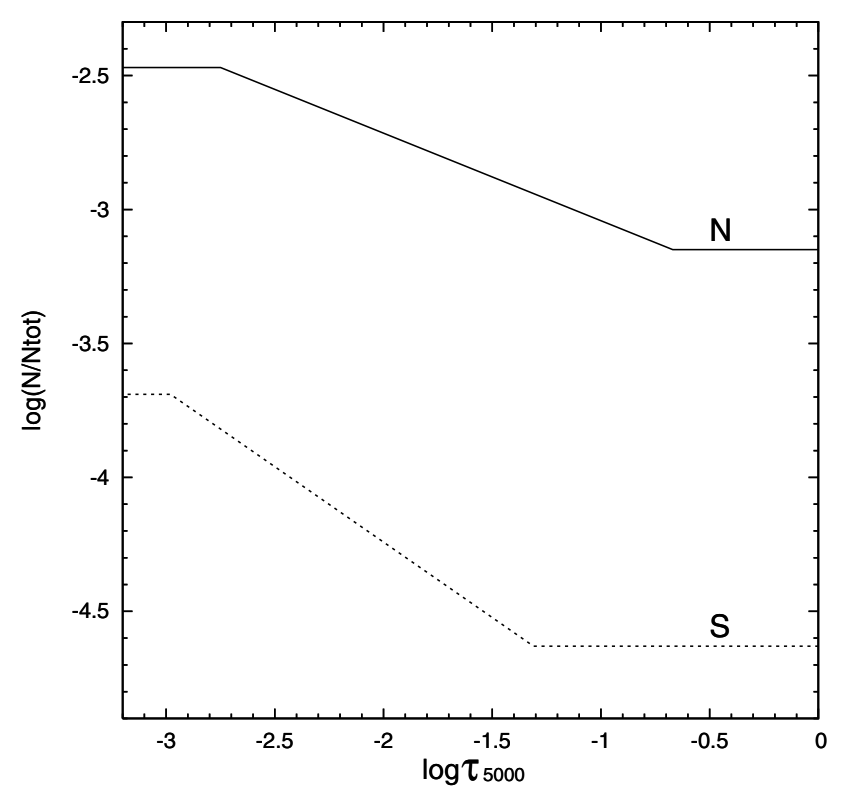

Fig. 3. Abundance stratification in the atmosphere of HD 135485 for nitrogen and sulfur.

explanation is that the abundances of $\mathrm{N}$ and $\mathrm{S}$ vary as a function of depth within the atmosphere. The detection of a similar trend of Fe abundance versus depth in the BHB star WF4-3085 in the globular cluster M 13 (Khalack et al. in preparation) suggests that the trends observed in HD 135485 for $\mathrm{N}$ and S, and possibly Fe, are real. These facts can be considered as further arguments in support of the efficiency of atomic diffusion in the atmosphere of HD 135485, and possibly in the atmospheres of BHB stars generally.

The study of chemical stratification in the atmospheres of BHB stars is a recent field of research. Previously Bonifacio et al. (1995) reported the possibility of helium stratification in the atmosphere of Feige 86. The results obtained here for nitrogen and sulfur stratification in HD 135485 are the first report of stratification of metal species in a BHB star. An extensive search for vertical stratification for a large number of chemical species in the atmospheres of BHB stars with a range of $T_{\text {eff }}$ would be very useful to place constraints on model atmospheres in which the atmospheric structure is calculated self-consistently with the stratification predicted by the diffusion phenomenon, such as those of Hui-Bon-Hoa, LeBlanc \& Hauschildt (2000). To obtain more precise results from observations pertaining to stratification of the elements, such models should be used since stratification can modify the atmospheric structure and thus the inferred stratification obtained by spectral analysis. For example, we found that the nitrogen stratification for HD 135485 is quite sensitive to the $T_{\text {eff }}$ used for the underlying model atmosphere.

Acknowledgements. This research was partially funded by the Natural Sciences and Engineering Research Council of Canada (NSERC). We thank the Réseau québécois de calcul de haute performance (RQCHP) for computing resources. GAW acknowledges support from the Academic Research Programme (ARP) of the Department of National Defence (Canada). BBB thanks the staff of McDonald Observatory for their assistance in collecting the CE data, and the National Research Council and Naval Research Laboratory for recent salary support. We are grateful to Dr. T.Ryabchikova for helpful discussions and suggestions.

\section{References}

Asplund, M., Grevesse, N., \& Sauval, A. J. 2005 in Cosmic Abundances as Records of Stellar Evolution and Nucleosynthesis, ed. T. G. Barnes, \& F. N. Bash, ASP Conf. Ser., 336, 25

Behr, B. B. 2003, ApJS, 149, 101

Behr, B. B., Djorgovski, S. G., \& Cohen, J. G., et al. 2000, ApJ, 528, 849

Bonifacio, P., Castelli, F., \& Hack, M. 1995, A\&AS, 110, 441

Donati, J.-F., Semel, M., Carter, B., et al. 1997, MNRAS, 291, 658

Donati, J.-F., Catala, C., Wade, G. A., et al. 1999, A\&AS, 134, 149

Dufton, P. L. 1973, A\&A, 28, 267

Deupree, R. G., \& Wallace, R. K. 1987, ApJ, 317, 724

Ferraro, F. R., Paltrinieri, B., Fusi Pecci, F., et al. 1998, ApJ, 500, 311

Fuhr, J. R., \& Wiese, W. L. 2006, J. Phys. Chem. Ref. Data, in press

Gies, D. R., \& Lambert, D. L. 1992, ApJ, 387, 673

Glaspey, J. W., Michaud, G., Moffat, A. F. J., \& Demers, S. 1989, ApJ, 339, 926

Grundhal, F., Catelan, M., Landsman, W. B., et al. 1999, ApJ, 524, 242

Hauschildt, P. H., Baron, E., \& Allard, F. 1997, ApJ, 483, 390

Hui-Bon-Hoa, A., LeBlanc, F., \& Hauschildt, P. H. 2000, ApJ, 535, L43

Khalack, V., Wade, G. 2006, A\&A, 450, 1157

Khalack, V., LeBlanc, F., et al., in preparation

Kupka, F., Piskunov, N. E., Ryabchikova, T. A., et al. 1999, A\&AS, 138, 119

Kurucz, R. L. 1993, CDROMs 13, 22, 23, SAO, Cambridge

Landstreet, J. D. 1988, ApJ, 326, 967

Landstreet, J. D. 1998, Contrib. Astron. Obs. Scalnaté Pleso, 27, 350

Lehner, N., Dufton, P. L., Lambert, D. L., et al. 2000, MNRAS 314, 199

McCarthy, J. K. 1990, in Proc. 2nd ESO/ST-ECF Data Analysis Workshop, (Garching: ESO), 119

McCarthy, J. K., Sandiford, B. A., Boyd, D., \& Booth, J. 1993, PASP, 105, 881

Peterson, R. C., Rood, R. T., \& Crocker, D. A. 1995, ApJ, 453, 214

Pickering, J. C., Johansson, S., \& Smith, P. L. 2001, A\&A, 377, 361

Press, W. H., Teukolsky, S. A., Vetterling, W. T., \& Flannery, B. P. 1992, Numerical recipes in C: the art of scientific computing, 2nd ed. (Cambridge University Press), 995

Raassen, A. J. J., \& Uylings, P. H. M. 1998, A\&A, 340, 300

Reader, J., Wiese, W. L., Martin, W. C., et al. 2002, NASA Laboratory Astrophysics Workshop, ed. F. Salama, Ref. Conf. Proc.: NASA/CP-200221186, 80

Recio-Blanco, A., Piotto, G., Aparicio, A., \& Renzini, A. 2004, A\&A, 417, 597

Ryabchikova, T. A., Piskunov, N. E., Stempels, H. C., Kupka, F., \& Weiss, W. W. 1999, in Proc. of the 6th International Colloquium om Atomic Spectra and Oscillator Strengths, Victoria BC, Physica Scripta T83, 162

Ryabchikova, T. A., Wade, G. A., \& LeBlanc, F. 2003, in Proc. of the Conference at University of North-West, Mmabatho, South Africa, ed. L. A. Balona, H. F. Henrichs, \& R. Medupe, ASP Conf. Proc., 305, 181

Ryabchikova, T., Leone, F., \& Kochukhov, O. 2005, A\&A, 438, 973

Schönberner, D. 1973, A\&A, 28, 443

Shortridge, K. 1993, The Figaro 2.4 Manual

Trundle, C., Dufton, P. L., Rolleston, W. R. J., et al. 2001, MNRAS, 328, 291

Wade, G. A., LeBlanc, F., Ryabchikova, T. A., \& Kudryavtsev, D. 2003, in Proc. of 210th IAU Symp on Modelling of Stellar Atmospheres, ed. N. Piskunov, W. W. Weiss, \& D. F. Gray, Published on behalf of the IAU by the Astronomical Society of the Pacific, D7

Wade, G. A., Bagnulo, S., Kochukhov, O., et al. 2001, A\&A, 374, 265 
V. R. Khalack et al.: Vertical abundance stratification in HD 135485, Online Material p 1

\section{Online Material}


V. R. Khalack et al.: Vertical abundance stratification in HD 135485, Online Material p 2

Table 2. List of spectral lines used for the abundance analysis.

\begin{tabular}{|c|c|c|c|c|}
\hline$\lambda, \AA$ & $\log g f$ & $E_{i}, \mathrm{~cm}^{-1}$ & $\log \gamma_{\mathrm{rad}}$ & Ref. \\
\hline \multicolumn{5}{|c|}{$\mathrm{HI}$} \\
\hline 4026.186 & -0.701 & 169086.76 & appx & NIST \\
\hline 4026.198 & -0.972 & 169086.84 & appx & NIST \\
\hline 4120.811 & -1.738 & 169086.76 & appx & NIST \\
\hline 4387.929 & -0.883 & 171134.89 & appx & NIST \\
\hline 4437.553 & -2.034 & 171134.89 & appx & NIST \\
\hline 4471.474 & -0.278 & 169086.76 & appx & NIST \\
\hline 4471.489 & -0.550 & 169086.84 & appx & NIST \\
\hline 4713.139 & -1.230 & 169086.76 & appx & NIST \\
\hline 4921.931 & -0.435 & 171134.89 & appx & NIST \\
\hline 5015.678 & -0.820 & 166277.44 & appx & NIST \\
\hline 5047.739 & -1.601 & 171134.89 & appx & NIST \\
\hline \multicolumn{5}{|c|}{$\mathrm{C}_{\mathrm{I}}$} \\
\hline 4371.367 & -1.962 & 61981.82 & appx & NIST \\
\hline 4771.742 & -1.866 & 60393.14 & appx & NIST \\
\hline 4932.039 & -1.658 & 61981.82 & appx & NIST \\
\hline 5380.337 & -1.616 & 61981.82 & appx & NIST \\
\hline 7113.179 & -0.773 & 69744.03 & appx & NIST \\
\hline 7115.168 & -0.934 & 69710.66 & appx & VALD \\
\hline 7116.988 & -0.907 & 69744.03 & appx & VALD \\
\hline 9061.433 & -0.346 & 60352.63 & appx & VALD \\
\hline 9078.288 & -0.581 & 60352.63 & appx & VALD \\
\hline 9088.515 & -0.429 & 60352.63 & appx & VALD \\
\hline 9111.809 & -0.298 & 60393.14 & appx & VALD \\
\hline 9658.431 & -0.280 & 60393.14 & appx & VALD \\
\hline \multicolumn{5}{|c|}{$\mathrm{C}_{\mathrm{II}}$} \\
\hline 3918.968 & -0.533 & 131724.37 & 8.98 & NIST \\
\hline 4267.261 & -0.584 & 145550.70 & 9.39 & NIST \\
\hline 4267.261 & 0.716 & 145550.70 & 9.39 & NIST \\
\hline 4313.106 & -0.373 & 186443.69 & appx & NIST \\
\hline 5132.947 & -0.211 & 166967.13 & 8.95 & NIST \\
\hline 5137.257 & -0.911 & 166967.13 & 8.95 & NIST \\
\hline 5139.174 & -0.707 & 166990.73 & 8.95 & NIST \\
\hline 5535.353 & -1.493 & 157234.07 & 8.94 & NIST \\
\hline 5648.072 & -0.424 & 166990.73 & 8.94 & VALD \\
\hline 5662.459 & -0.249 & 167035.71 & 8.94 & VALD \\
\hline 5891.597 & -0.440 & 145549.27 & appx & VALD \\
\hline 6098.512 & 0.226 & 182043.41 & appx & VALD \\
\hline 6779.940 & 0.025 & 166990.73 & 8.93 & NIST \\
\hline 6783.908 & 0.304 & 167035.71 & 8.93 & VALD \\
\hline 7231.337 & 0.043 & 131724.37 & appx & VALD \\
\hline 7237.166 & -0.656 & 131735.52 & appx & VALD \\
\hline \multicolumn{5}{|c|}{ NI } \\
\hline 6482.699 & -0.510 & 94881.82 & appx & VALD \\
\hline 7442.298 & -0.385 & 83317.83 & 8.64 & VALD \\
\hline 7898.982 & 0.024 & 99663.43 & appx & VALD \\
\hline 7899.284 & -0.911 & 99663.91 & appx & VALD \\
\hline \multicolumn{5}{|c|}{ N II } \\
\hline 4447.030 & 0.228 & 164610.76 & 9.16 & NIST \\
\hline 4507.556 & -0.817 & 166678.64 & 9.33 & VALD \\
\hline 4601.478 & -0.428 & 148940.17 & 9.23 & VALD \\
\hline 4613.868 & -0.665 & 148940.17 & 9.23 & VALD \\
\hline 4621.396 & -0.514 & 148940.17 & 9.23 & VALD \\
\hline 4630.539 & 0.094 & 149076.52 & 9.15 & VALD \\
\hline 4779.722 & -0.587 & 166521.69 & 9.58 & VALD \\
\hline 4803.287 & -0.113 & 166678.64 & 9.58 & VALD \\
\hline 4987.376 & -0.555 & 168892.21 & 9.34 & VALD \\
\hline 4994.367 & -0.069 & 168892.21 & 9.34 & VALD \\
\hline 5001.135 & 0.263 & 166521.69 & 8.45 & VALD \\
\hline 5001.475 & 0.439 & 166582.45 & 8.45 & VALD \\
\hline 5005.150 & 0.592 & 166678.64 & 8.25 & VALD \\
\hline 5007.333 & 0.171 & 168892.21 & 9.33 & VALD \\
\hline 5010.621 & -0.606 & 148940.17 & 9.22 & VALD \\
\hline 5025.659 & -0.546 & 166678.64 & 8.43 & VALD \\
\hline 5480.050 & -0.756 & 170666.23 & 9.34 & VALD \\
\hline 5676.020 & -0.368 & 148908.59 & 9.14 & VALD \\
\hline
\end{tabular}


V. R. Khalack et al.: Vertical abundance stratification in HD 135485, Online Material p 3

Table 2. continued.

\begin{tabular}{|c|c|c|c|c|}
\hline$\lambda, \AA$ & $\log g f$ & $E_{i}, \mathrm{~cm}^{-1}$ & $\log \gamma_{\mathrm{rad}}$ & Ref. \\
\hline 5679.554 & 0.250 & 149076.52 & 9.13 & VALD \\
\hline 5686.212 & -0.549 & 148940.17 & 9.22 & VALD \\
\hline 5941.650 & 0.313 & 170666.23 & 9.59 & VALD \\
\hline \multicolumn{5}{|c|}{ O I } \\
\hline 4368.242 & -1.964 & 76794.98 & 8.76 & NIST \\
\hline 5329.681 & -1.473 & 86627.78 & 7.54 & NIST \\
\hline 5329.690 & -1.268 & 86627.78 & 7.54 & NIST \\
\hline 5330.735 & -1.570 & 86631.45 & 7.55 & VALD \\
\hline 5330.741 & -0.983 & 86631.45 & 7.55 & VALD \\
\hline 6155.961 & -1.363 & 86625.76 & 7.60 & NIST \\
\hline 6155.971 & -1.011 & 86625.76 & 7.61 & NIST \\
\hline 6155.989 & -1.120 & 86625.76 & 7.61 & NIST \\
\hline 6158.172 & -0.995 & 86631.45 & 7.62 & NIST \\
\hline 6158.177 & -0.409 & 86631.45 & 7.61 & NIST \\
\hline 6454.444 & -1.066 & 86627.78 & 7.66 & NIST \\
\hline 7001.922 & -1.011 & 86630.59 & 7.93 & NIST \\
\hline 7002.230 & -0.741 & 86631.15 & 7.93 & NIST \\
\hline 7156.701 & 0.288 & 102662.03 & 8.76 & NIST \\
\hline 7254.448 & -1.054 & 88631.15 & 7.59 & NIST \\
\hline 7254.531 & -1.753 & 88631.30 & 7.59 & NIST \\
\hline 8446.758 & 0.014 & 76794.98 & 8.77 & NIST \\
\hline 8820.423 & 0.379 & 102662.03 & 8.74 & NIST \\
\hline \multicolumn{5}{|c|}{ O II } \\
\hline 4069.882 & 0.344 & 206786.2978 .48 & NIST & \\
\hline 4072.157 & 0.552 & 206877.86 & 8.50 & NIST \\
\hline 4119.216 & 0.452 & 208484.20 & 9.09 & NIST \\
\hline 4414.899 & 0.172 & 189068.51 & 9.46 & NIST \\
\hline 4641.810 & 0.055 & 185340.58 & 8.96 & NIST \\
\hline 4649.135 & 0.308 & 185499.12 & 8.96 & NIST \\
\hline 4650.838 & -0.362 & 185235.28 & 8.96 & NIST \\
\hline 4661.632 & -0.278 & 185340.58 & 8.96 & NIST \\
\hline 4676.235 & -0.394 & 185499.12 & 8.96 & NIST \\
\hline \multicolumn{5}{|c|}{$\mathrm{NeI}$} \\
\hline 7488.871 & 0.167 & 148257.79 & 7.95 & NIST \\
\hline 7535.774 & 0.040 & 148257.79 & 8.12 & NIST \\
\hline 8377.608 & 0.680 & 149657.04 & 8.01 & NIST \\
\hline 8654.383 & 0.544 & 150858.51 & 8.03 & NIST \\
\hline \multicolumn{5}{|c|}{$\mathrm{NaI}$} \\
\hline 5688.205 & -0.452 & 16973.37 & appx & NIST \\
\hline 8183.255 & 0.237 & 16956.17 & appx & NIST \\
\hline \multicolumn{5}{|c|}{$\overline{M g} \mathbf{I}$} \\
\hline 5172.684 & -0.393 & 21870.46 & 7.99 & NIST \\
\hline \multicolumn{5}{|c|}{$\overline{\mathrm{Mg} \text { II }}$} \\
\hline 4390.572 & -0.523 & 80650.02 & appx & NIST \\
\hline 4433.988 & -0.907 & 80650.02 & appx & NIST \\
\hline 7877.054 & 0.391 & 80619.50 & appx & NIST \\
\hline 7896.366 & 0.643 & 80650.02 & appx & NIST \\
\hline \multicolumn{5}{|c|}{$\overline{\mathrm{Al}}$ II } \\
\hline 4663.046 & -0.284 & 85481.35 & 7.99 & VALD \\
\hline 5593.300 & 0.410 & 106920.56 & appx & VALD \\
\hline 6243.203 & -0.080 & 105470.93 & appx & VALD \\
\hline 6243.367 & 0.670 & 105470.93 & appx & VALD \\
\hline 6823.390 & -0.140 & 105441.50 & appx & VALD \\
\hline 6837.130 & 0.080 & 105470.93 & appx & VALD \\
\hline 6920.343 & -0.160 & 105470.93 & appx & VALD \\
\hline 7471.410 & 0.780 & 110089.83 & appx & VALD \\
\hline \multicolumn{5}{|c|}{$\mathrm{Al}$ III } \\
\hline 4512.565 & 0.410 & 143633.38 & appx & VALD \\
\hline 4529.189 & 0.660 & 143713.50 & appx & VALD \\
\hline 5696.604 & 0.230 & 126164.05 & appx & VALD \\
\hline 5722.730 & -0.070 & 126164.05 & appx & VALD \\
\hline \multicolumn{5}{|c|}{ Si II } \\
\hline 4128.054 & 0.306 & 79338.50 & 9.44 & NIST \\
\hline 4130.872 & -0.840 & 79355.02 & 9.44 & NIST \\
\hline 4130.894 & 0.464 & 79355.02 & 9.44 & NIST \\
\hline 4621.722 & -0.390 & 101024.35 & 9.01 & NIST \\
\hline 5055.984 & 0.440 & 81251.32 & 9.04 & NIST \\
\hline
\end{tabular}


V. R. Khalack et al.: Vertical abundance stratification in HD 135485, Online Material p 4

Table 2. continued.

\begin{tabular}{|c|c|c|c|c|}
\hline$\lambda, \AA$ & $\log g f$ & $E_{i}, \mathrm{~cm}^{-1}$ & $\log \gamma_{\mathrm{rad}}$ & Ref. \\
\hline 5466.849 & -1.340 & 101024.35 & 9.02 & NIST \\
\hline 5466.894 & -0.030 & 101024.35 & 9.02 & NIST \\
\hline 5669.563 & 0.690 & 114530.87 & 8.07 & VALD \\
\hline 5868.444 & 0.400 & 117176.37 & 8.04 & VALD \\
\hline 5957.560 & -0.350 & 81191.34 & 8.82 & NIST \\
\hline 7849.618 & -0.810 & 101024.35 & 9.04 & NIST \\
\hline 7849.722 & 0.490 & 101024.35 & 9.04 & NIST \\
\hline \multirow{3}{*}{$\begin{array}{l}4552.622 \\
4574.757\end{array}$} & & Si III & & \\
\hline & 0.292 & 153377.05 & 9.08 & NIST \\
\hline & -0.406 & 153377.05 & 9.08 & NIST \\
\hline \multicolumn{5}{|c|}{$\overline{\mathrm{P} \text { II }}$} \\
\hline 4420.712 & -0.478 & 88893.22 & 8.29 & NIST \\
\hline 4499.230 & 0.377 & 107922.93 & 8.27 & NIST \\
\hline 4602.069 & 0.799 & 103667.86 & 8.25 & NIST \\
\hline 5296.077 & -0.134 & 87124.60 & 8.13 & NIST \\
\hline 5344.729 & -0.390 & 86597.55 & 8.12 & NIST \\
\hline 5425.880 & 0.241 & 87124.60 & 8.11 & NIST \\
\hline 6043.080 & 0.384 & 87124.60 & 8.01 & NIST \\
\hline 6459.945 & 0.101 & 88192.13 & 7.96 & NIST \\
\hline \multicolumn{5}{|c|}{ S II } \\
\hline 4028.750 & 0.000 & 128599.16 & 8.60 & NIST \\
\hline 4032.768 & 0.240 & 131028.85 & 9.38 & NIST \\
\hline 4142.259 & 0.240 & 127825.08 & 8.62 & NIST \\
\hline 4153.068 & 0.620 & 128233.20 & 8.52 & NIST \\
\hline 4162.665 & 0.780 & 128599.16 & 8.62 & NIST \\
\hline 4165.100 & 0.060 & 140230.10 & 9.21 & NIST \\
\hline 4168.384 & -0.160 & 127976.34 & 8.63 & NIST \\
\hline 4217.182 & -0.150 & 128599.16 & 8.49 & NIST \\
\hline 4257.379 & 0.360 & 140750.34 & 9.24 & NIST \\
\hline 4267.762 & 0.290 & 129858.18 & 8.71 & NIST \\
\hline 4269.725 & 0.080 & 129787.83 & 8.66 & VALD \\
\hline 4278.506 & -0.114 & 129787.83 & 8.66 & NIST \\
\hline 4282.593 & -0.010 & 129858.18 & 8.64 & NIST \\
\hline 4294.402 & 0.549 & 130134.16 & 8.62 & VALD \\
\hline 4432.368 & -0.470 & 127976.34 & 8.68 & NIST \\
\hline 4456.382 & -0.560 & 127825.08 & 8.74 & VALD \\
\hline 4463.581 & 0.134 & 128599.16 & 8.73 & VALD \\
\hline 4486.634 & -0.400 & 127976.34 & 8.76 & NIST \\
\hline 4716.271 & 0.410 & 109831.59 & 8.88 & NIST \\
\hline 4792.007 & 0.306 & 130134.16 & 8.74 & VALD \\
\hline 4815.552 & 0.090 & 110268.60 & 8.87 & NIST \\
\hline 4819.445 & -0.490 & 129787.83 & 8.66 & NIST \\
\hline 4819.626 & -0.220 & 130641.11 & 8.93 & NIST \\
\hline 4835.846 & -0.990 & 129858.18 & 8.64 & VALD \\
\hline 4885.648 & -0.610 & 112937.57 & 8.75 & NIST \\
\hline 4900.513 & -0.490 & 129858.18 & 8.72 & VALD \\
\hline 4901.277 & -0.460 & 130134.16 & 8.66 & VALD \\
\hline 4917.198 & -0.320 & 112937.57 & 8.76 & NIST \\
\hline 4925.343 & -0.235 & 109560.69 & 8.86 & VALD \\
\hline 4942.473 & -0.960 & 109560.69 & 8.87 & NIST \\
\hline 4991.969 & -0.246 & 109831.59 & 8.85 & VALD \\
\hline 5009.567 & -0.280 & 109831.59 & 8.86 & NIST \\
\hline 5014.042 & 0.030 & 113461.54 & 8.75 & VALD \\
\hline 5027.203 & -0.710 & 105599.06 & 8.75 & NIST \\
\hline 5032.434 & 0.270 & 110268.60 & 8.85 & NIST \\
\hline 5103.332 & -0.275 & 110268.60 & 8.84 & VALD \\
\hline 5142.322 & -0.820 & 106044.24 & 8.70 & NIST \\
\hline 5201.027 & 0.090 & 121528.72 & 9.16 & NIST \\
\hline 5320.723 & 0.490 & 121530.02 & 9.09 & NIST \\
\hline 5428.655 & -0.120 & 109560.69 & 8.88 & VALD \\
\hline 5453.855 & 0.557 & 110268.60 & 8.85 & VALD \\
\hline 5509.705 & -0.117 & 109831.59 & 8.88 & VALD \\
\hline 5564.958 & -0.320 & 110268.60 & 8.86 & NIST \\
\hline 5616.633 & -0.640 & 110177.02 & 8.60 & NIST \\
\hline 5639.977 & 0.330 & 113461.54 & 8.75 & VALD \\
\hline 5647.020 & 0.110 & 112937.57 & 8.75 & VALD \\
\hline 5819.254 & -0.760 & 113461.54 & 8.76 & NIST \\
\hline
\end{tabular}


V. R. Khalack et al.: Vertical abundance stratification in HD 135485, Online Material p 5

Table 2. continued.

\begin{tabular}{|c|c|c|c|c|}
\hline$\lambda, \AA$ & $\log g f$ & $E_{i}, \mathrm{~cm}^{-1}$ & $\log \gamma_{\mathrm{rad}}$ & Ref. \\
\hline 6305.482 & -0.190 & 114279.33 & 8.28 & NIST \\
\hline \multirow[b]{2}{*}{4794.556} & & $\mathrm{Cl}$ II & & \multirow[b]{2}{*}{ VALD } \\
\hline & 0.455 & 107879.66 & appx & \\
\hline & & Ar II & & \\
\hline 4426.001 & 0.158 & 135086.00 & appx & NIST \\
\hline 4609.567 & 0.304 & 148842.47 & appx & NIST \\
\hline 4806.020 & 0.210 & 134241.74 & appx & NIST \\
\hline 4879.863 & 0.296 & 138243.64 & appx & NIST \\
\hline & & Ti II & & \\
\hline 4300.049 & -0.490 & 9518.06 & 8.47 & VALD \\
\hline 4443.794 & -0.700 & 8710.44 & 8.20 & VALD \\
\hline 4501.273 & -0.760 & 8997.71 & 8.20 & VALD \\
\hline 4563.761 & -0.790 & 9850.90 & 8.22 & VALD \\
\hline 4571.985 & -0.230 & 12676.97 & 8.37 & VALD \\
\hline & & Cr II & & \\
\hline 4558.650 & -0.449 & 32854.31 & 8.41 & VALD, R\&U \\
\hline 4558.783 & -2.530 & 32859.07 & 8.41 & VALD, R\&U \\
\hline 4588.199 & -0.627 & 32836.68 & 8.41 & VALD, R\&U \\
\hline 4616.539 & -2.020 & 91576.30 & 8.92 & VALD, R\&U \\
\hline 4616.629 & -1.361 & 32844.76 & 8.41 & VALD, R\&U \\
\hline 4634.070 & -0.990 & 32844.76 & 8.41 & VALD, R\&U \\
\hline 5237.329 & -1.350 & 32854.31 & 8.40 & VALD, R\&U \\
\hline 5237.329 & -0.740 & 86785.36 & 8.85 & VALD, R\&U \\
\hline & & Fe II & & \\
\hline 4044.012 & -2.671 & 44929.55 & 8.70 & $R \& U$ \\
\hline 4048.832 & -2.381 & 44917.07 & 8.73 & $\mathrm{R} \& \mathrm{U}$ \\
\hline 4048.931 & -2.779 & 73603.50 & 8.99 & $\mathrm{R} \& \mathrm{U}$ \\
\hline 4173.461 & -2.617 & 20830.58 & 8.61 & VALD \\
\hline $4173.473^{*}$ & -2.185 & 24333.78 & 8.51 & VALD, F\&W \\
\hline 4178.862 & -2.535 & 20830.58 & 8.49 & $R \& U$ \\
\hline 4233.172 & -1.947 & 20830.58 & 8.61 & $R \& U$ \\
\hline 4258.154 & -3.478 & 21812.06 & 8.48 & $R \& U$ \\
\hline 4273.326 & -3.303 & 21812.06 & 8.62 & $\mathrm{R} \& \mathrm{U}$ \\
\hline 4296.572 & -2.933 & 21812.06 & 8.49 & $R \& U$ \\
\hline 4303.176 & -2.511 & 21812.06 & 8.61 & $\mathrm{R} \& \mathrm{U}$ \\
\hline 4351.769 & -2.216 & 21812.06 & 8.61 & $\mathrm{R} \& \mathrm{U}$ \\
\hline 4385.381 & -2.581 & 22409.85 & 8.62 & $\mathrm{R} \& \mathrm{U}$ \\
\hline 4416.830 & -2.602 & 22409.85 & 8.61 & $R \& U$ \\
\hline 4461.706 & -2.065 & 50212.83 & 8.62 & $R \& U$ \\
\hline 4489.183 & -2.971 & 22810.36 & 8.49 & $\mathrm{R} \& \mathrm{U}$ \\
\hline 4491.405 & -2.755 & 23031.30 & 8.48 & $R \& U$ \\
\hline 4508.214 & -2.589 & 63948.79 & 9.01 & $\mathrm{R} \& \mathrm{U}$ \\
\hline 4508.288 & -2.349 & 23031.30 & 8.62 & $R \& U$ \\
\hline 4515.339 & -2.540 & 22939.36 & 8.49 & $\mathrm{R} \& \mathrm{U}$ \\
\hline 4522.634 & -2.169 & 22939.36 & 8.61 & $R \& U$ \\
\hline 4541.524 & -2.973 & 23031.30 & 8.61 & $R \& U$ \\
\hline 4555.893 & -2.421 & 22810.36 & 0.85 & $R \& U$ \\
\hline 4576.340 & -2.976 & 22939.36 & 8.61 & $\mathrm{R} \& \mathrm{U}$ \\
\hline 4582.835 & -3.224 & 22939.36 & 8.49 & $R \& U$ \\
\hline 4583.837 & -1.867 & 22637.21 & 8.61 & $\mathrm{R} \& \mathrm{U}$ \\
\hline 4596.015 & -1.956 & 50212.83 & 8.74 & $R \& U$ \\
\hline 4629.339 & -2.478 & 22637.21 & 8.48 & $R \& U$ \\
\hline 4635.316 & -1.578 & 48039.09 & 8.73 & $\mathrm{R} \& \mathrm{U}$ \\
\hline 4731.453 & -3.127 & 23317.63 & 8.61 & $R \& U$ \\
\hline 4951.584 & 0.198 & 83136.49 & 8.96 & $\mathrm{R} \& \mathrm{U}$ \\
\hline $5001.864^{*}$ & 0.010 & 31302.42 & 7.97 & VALD, F\&W \\
\hline 5001.959 & 0.916 & 82978.68 & 8.93 & $R \& U$ \\
\hline 5004.195 & 0.504 & 82853.66 & 8.93 & $R \& U$ \\
\hline 5018.440 & -1.345 & 23317.63 & 8.49 & $\mathrm{R} \& \mathrm{U}$ \\
\hline 5030.630 & 0.433 & 82978.68 & 9.02 & $R \& U$ \\
\hline 5032.712 & 0.107 & 83812.32 & 8.99 & $\mathrm{R} \& \mathrm{U}$ \\
\hline 5035.708 & 0.639 & 82978.68 & 8.94 & $R \& U$ \\
\hline 5061.718 & 0.290 & 83136.49 & 9.05 & $\mathrm{R} \& \mathrm{U}$ \\
\hline 5070.899 & 0.275 & 83136.49 & 9.02 & $\mathrm{R} \& \mathrm{U}$ \\
\hline 5075.764 & 0.168 & 84326.91 & 8.94 & $R \& U$ \\
\hline 5097.271 & 0.315 & 83713.54 & 8.94 & $R \& U$ \\
\hline 5144.355 & 0.295 & 84424.37 & 8.99 & $R \& U$ \\
\hline
\end{tabular}


V. R. Khalack et al.: Vertical abundance stratification in HD 135485, Online Material p 6

Table 2. continued.

\begin{tabular}{crccl}
\hline \hline$\lambda, \AA$ & $\log g f$ & $E_{i}, \mathrm{~cm}^{-1}$ & $\log \gamma_{\text {rad }}$ & Ref. \\
\hline 5149.465 & 0.544 & 84266.56 & 8.92 & R\&U \\
5169.033 & -1.250 & 23317.63 & 8.49 & R\&U \\
5197.568 & -2.105 & 83865.63 & 9.05 & VALD \\
5197.577 & -2.348 & 26055.42 & 8.48 & R\&U \\
5199.122 & 0.107 & 83713.54 & 9.02 & R\&U \\
5216.854 & 0.480 & 84710.68 & 8.96 & R\&U \\
5216.863 & 0.659 & 84527.78 & 8.93 & R\&U \\
5227.323 & 0.191 & 84844.83 & 8.93 & R\&U \\
5227.481 & 0.834 & 84296.83 & 8.91 & R\&U \\
5234.615 & -2.385 & 84266.56 & 8.98 & R\&U \\
5234.625 & -2.279 & 25981.63 & 8.49 & R\&U \\
5247.952 & 0.550 & 84938.18 & 8.95 & R\&U \\
5260.259 & 1.069 & 84035.01 & 8.91 & VALD \\
5272.397 & -2.009 & 48039.09 & 8.69 & R\&U \\
5276.002 & -2.213 & 25805.33 & 8.49 & R\&U \\
5284.109 & -3.195 & 23317.63 & 8.53 & R\&U \\
5291.666 & 0.556 & 84527.78 & 9.00 & R\&U \\
5316.225 & 0.340 & 84035.14 & 8.92 & R\&U \\
5316.615 & -2.014 & 25428.78 & 8.48 & R\&U \\
5316.784 & -2.783 & 25981.63 & 8.61 & R\&U \\
5339.585 & 0.540 & 84296.83 & 8.91 & R\&U \\
5387.063 & 0.522 & 84863.35 & 8.89 & R\&U \\
5429.988 & 0.438 & 85462.86 & 8.93 & R\&U \\
5482.308 & 0.405 & 85184.73 & 8.98 & R\&U \\
5506.195 & 0.849 & 84863.35 & 8.89 & R\&U \\
5780.128 & 0.413 & 86124.30 & 8.96 & R\&U \\
5783.630 & 0.362 & 86416.33 & 8.97 & R\&U \\
5902.825 & 0.420 & 86416.33 & 9.09 & R\&U \\
5961.705 & 0.678 & 86124.30 & 9.20 & R\&U \\
\hline & & Sr II & & \\
4077.709 & 0.167 & 0.00 & $a p p x$ & VALD \\
\hline & & & &
\end{tabular}

